(cSLE) patients, defined as diagnosis $<18$ years of age, vs. adult-onset SLE (aSLE), using logistic regression, and adjusted for the same covariates. We conducted sensitivity analyses where we stratified GWAS by cSLE and aSLE, then meta-analyzed results using inverse variance weighting, as well as ancestry-stratified analyses (Europeans, East Asians, Africans, Amerindians and Admixed).

Results Our cohort included 1489 patients, 761 (51\%) cSLE, $1306(88 \%)$ female. Median age at diagnosis was 17.7 years (IQR 14, 31) in the total cohort, 14.1 years (IQR 11.8, 15.8) in cSLE, and 31.2 years (IQR 24.7, 42) in aSLE. In the total cohort, 576 (39\%) were of European ancestry, 278 (19\%) East Asian, and 253 (17\%) Admixed. We included 11.7M SNPs in GWAS. In the age of SLE diagnosis GWAS, 2 loci on chr16 were genome-wide significantly associated with younger age at diagnosis (top SNP rs11641349, Beta $-0.03 \mathrm{y}$, SE $0.15 \mathrm{y}$, $\mathrm{P}=4.33 \times 10^{-8}$, MAF 0.2). Both SNPs were intronic to CCDC113, a component of centriolar satellites. These loci were also the most significant in the GWAS of cSLE (top SNP rs16959933, OR 1.75 [95\% CI: 1.43, 2.14, P=5.45 x10-8]). Sensitivity analyses showed similar results, yet they did not reach genome-wide significance with top SNPs rs11641349 $\left(\mathrm{P}=3.84 \times 10^{-7}\right)$ and $\operatorname{rs} 16959933\left(\mathrm{P}=4.50 \times 10^{-7}\right)$ in the age group model, and rs11641349 $\left(\mathrm{P}=3.52 \times 10^{-7}\right)$ and $\mathrm{rs} 16959933$ $\left(\mathrm{P}=4.22 \times 10^{-7}\right)$ in the ancestry model.

Conclusion In our multiethnic cSLE and aSLE cohort, we identified genome-wide significant loci associated with age at diagnosis and cSLE risk, intronic to CCDC113. Our study requires independent validation.

\section{GENETIC PREDISPOSITION TO LUPUS ACROSS ANCESTRIES HAS $>300$ SEPARABLE GENETIC CONTRIBUTIONS: WHAT WE KNOW TODAY}

${ }^{1}$ Viktoryia Laurynenka, 1,2Leah C Kottyan, 1,2 Matthew T Weirauch, 1,2,3 Kenneth M Kaufman, ${ }^{3} J o h n$ B Harley*. 'Center for Autoimmune Genomics and Etiology, Cincinnati Children's Hospital Medical Center, Cincinnati, Ohio, USA; ${ }^{2}$ University of Cincinnati College of Medicine, Cincinnati, Ohio, USA; ${ }^{3}$ Research Service, US Department of Veterans Affairs (USDVA) Medical Center, Cincinnati, Ohio, USA

\subsection{6/lupus-2021-lupus21century.85}

Background Systemic lupus erythematosus (SLE) is a complex disease caused by interplay of genetic predisposition and environmental triggers. Strong genetic component in lupus supported by familial clustering, where $10-12 \%$ of SLE patients have an affected first-degree relative, and high concordance rates (24-69\%) in monozygotic twins compared to dizygotic twins and non-twin siblings (2-9\%). In the majority of SLE cases, excluding rare monogenic forms, the genetic components are composed of many polymorphisms with small effects, acting additively, which explain about $44 \%$ to $66 \%$ of the disease etiology. The prevalence and severity of the disease differ across populations, where Africans, Hispanics and East Asians having three to four times higher incidence compared to Europeans, which may be partially explained by genetic differences, as we show here. We summarized all the genetic findings in candidate gene and genome wide association studies (GWAS) for non-monogenic forms of lupus across different populations now available in the medical literature.

Methods A literature search for GWAS and candidate gene studies in SLE was done in the electronic article database PubMed (www.ncbi.nlm.nih.gov/pubmed), NHGRI-EBI GWAS catalog and the references of selected original publications and review articles. We included 127 studies that reported SLE-associated polymorphisms reaching the threshold of significance $\leq 5 \times 10^{-8}$. Variants in linkage disequilibrium (LD) with $\mathrm{r}^{2} \geq 0.8$ were grouped into the same loci. The leading variant of the locus was defined as a variant with lowest $\mathrm{p}$ value. The criteria for the independence of loci were $\mathrm{r}^{2}<0.2$ between leading variants or literature support for there being two (or more) distinguishable contributions to genetic risk.

Results In total we found 730 polymorphisms with $\mathrm{p}$ value between $5 \times 10^{-8}$ and $2.2 \times 10^{-298}$ associated with SLE in Europeans (EU), Asians (AS), African-American (AA) and Mixed Americans/Hispanics (MA). Reported odds ratio (OR) varied between 1.1 and 5, but the majority of the associations are weak effects $(\sim 77 \%$ have $\mathrm{OR}<1.5$ while $\sim 7.7 \%$ have an $\mathrm{OR} \geq 2$ ). These variants we grouped into 315 independent loci: 106 loci in EU, 216 loci in AS, 11 loci in AA, 18 loci in MA and 28 loci was reported only in multiancestral group. Many loci are ancestry specific: 174 (80.56\%) loci are found to date only in AS, 60 (56.60\%) loci only in EU, 3 (27.27\%) loci only in AA, and $3(16.67 \%)$ loci only in MA (figure 1). This finding may be explained by the different allele frequencies in the populations studied. This population specificity of the disease loci could influence on SLE prevalence and be a source for heterogeneity of symptoms and disease severity across populations. Identifying the true causal variants and predicting their function is not a trivial task, as genetic variants are in linkage blocks; therefore, the variant with lowest $\mathrm{p}$ value may not be causal. Among 730 SLE associated polymorphisms only $21(4.52 \%)$ lead to amino acid change, 484 $(66.3 \%)$ lay within gene coding region of 272 genes and the rest are intergenic, which suggests that the majority of SLE associations affect gene regulation, instead of protein sequence. These variants may regulate gene or genes near or far, making the identification of their targets that are involved in mechanisms that change lupus risk a major challenge. The pathway analysis for the 272 genes intersected by SLE variants shows that they are involved in immune processes including responses to pathogens and transcription (MHC class II receptor activity, toll-like receptor signaling, response to cytokines and regulation of their production, immune cell activation and proliferation, DNA binding, transcription regulation, EpsteinBarr virus infection and many others).

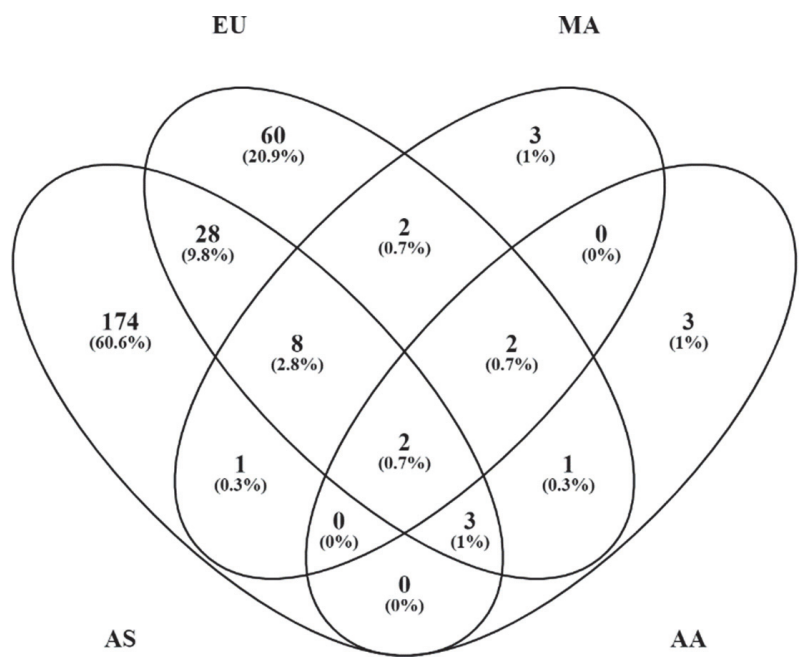

Abstract 1502 Figure 1 
Conclusions GWAS and candidate gene studies discovered more than 300 lupus associated loci that explain the more than half of heritability in SLE, presumably leaving many SLE loci to be discovered in the future. Extensive studies have been performed in East Asians and Europeans with studies involving fewer subjects mean that we know less about SLE genetics in other populations. Many SLE associated variants are ancestry specific that have the potential to give distinction to clinical manifestations and disease prevalence when comparing ancestries. The great majority of SLE associated variants are located in non-coding regions; therefore, they more likely affect gene regulation, making this mechanism the key for understanding SLE genetics and pathogenesis. Surrogate candidate genes potentially affected by SLE associated polymorphisms appear to be mainly involved in immune processes and the regulation of transcription.

Acknowledgments Support is appreciated from US Department of Veterans Affairs Merit Award (I01 BX001834), and the National Institutes of Health (R01 AI24717 \& $\quad$ U01 AI130830).

\section{HIGH-THROUGHPUT IDENTIFICATION OF REGULATORY FUNCTIONAL SNPS ASSOCIATED WITH SYSTEMIC LUPUS ERYTHEMATOSUS}

${ }^{1}$ Qiang Wang, ${ }^{2}$ Marta Martínez Bonet, ${ }^{3}$ Matthew Weirauch, ${ }^{4}$ Peter A Nigrovic*. ${ }^{1}$ Boston Children's Hospital/Harvard Medical School, USA; ${ }^{2}$ Instituto de Investigación Sanitaria Gregorio Marañón, Spain; ${ }^{3}$ Cincinnati Children's Hospital Medical Center/University of Cincinnati, USA; ${ }^{4}$ Boston Children's Hospital/Brigham and Women's Hospital/Harvard Medical School, USA

\subsection{6/lupus-2021-lupus21century.86}

Background Systemic lupus erythematosus (SLE) is a disease involves the complex interplay of many genes, reflected in more than one hundred loci linked with disease risk by genome-wide association studies (GWAS). Decoding GWAS is therefore a promising strategy to identify novel drug targets in SLE. However, most of the identified disease-associated hits are noncoding single-nucleotide polymorphisms (SNPs), and cannot be distinguished from others that reside incidentally within risk loci. To address this longstanding challenge of finding the real regulatory functional SNPs (fSNPs) from among GWAS hits in SLE, we utilized an unbiased highthroughput screen method.

Methods From 5 GWAS for SLE (Gateva, Sandling et al. 2009, Bentham, Morris et al. 2015, Armstrong et al. 2015, Morris, Sheng et al. 2016, Langefeld, Ainsworth et al. 2017), 87 disease associated SNPs were chosen as lead SNPs and SNPs in linkage disequilibrium $(\mathrm{LD})\left(\mathrm{R}^{2}>0.8\right)$ with them are also included as the screening library. In total of 2176 SNPs were screened by three different high-throughput methods, SNP-seq (Li, Martinez-Bonet et al. 2018), H3K4me3 epigenetic modification (Trynka, Sandor et al. 2013), and Combined Annotation Dependent Depletion (CADD) (Rentzsch, Witten et al. 2018). Top candidates from the screening were further tested for regulatory function by electrophoretic mobility shift assay (EMSA) and luciferase reporter assay to define the final fSNPs candidates. Through bioinformatics binding motif prediction and mass spectrometry after oligo pulldown, transcriptional factors (TF) that might binds to the fSNPs were prioritized for validation by CHIP-qPCR, Western blot for oligo pulldown assay, and supershift. The association between selected fSNPs and the risk gene/associated gene was measured using genetic modified of the Daudi B cell line (CRISPRhomology directed repair). The association between the TF and the associated gene was measured by TF CRISPR knockout Daudi B cells.

Results Fifty-four candidate fSNPs from 2176 SNPs were found to be possible regulatory variants and tested for regulatory function. After EMSA, 9 SNPs showed allele-specific binding to proteins from both BL2 cells (B cell line) and PBMC nuclear extract. Six out of these 9 SNPs showed allelic differential gene expression in luciferase reporter assay in a B cell line (Daudi). After bioinformatics predictions as well as mass spectrometry for oligo pulldown assay, two fSNPs (rs2297550 and rs9907966) were found to be able to bind to transcriptional factor IKZF1 and YBX1 in B cells respectively. Specifically, IKZF1 prefers to bind to $G$ allele (risk allele) of rs2297550 and YBX1 prefers to bind to A allele (reference allele) of rs9907966. SNP rs2297550 was further analyzed for its association with the risk gene IKBKE and found out that the homozygous of risk $G$ allele is associated with lower IKBKE expression in $\mathrm{B}$ cells. Considering the role of IKBKE in preventing DNA damage induced cell death, the deficiency of IKBKE plausibly increases SLE risk. Through CRISPR knockout, we found out that the deficiency of TF IKZF1 leads to increased IKBKE expression. These data suggest a plausible mechanism which the risk allele of rs2297550 binds to IKZF1 to suppress the expression of IKBKE in B cells, therefore increase SLE risk.

Conclusions Our unbiased high-throughput screening for SLE GWAS hits followed by a step-wise validation leads to the identification of real functional regulatory fSNPs that are capable of binding to transcriptional factors and regulate gene expression, which establish a working model to bridge the gap between SLE GWAS and disease mechanism.

Acknowledgments This work was funded by a Target Identification in Lupus grant from the Lupus Research Alliance.

\section{THE INFLUENCE OF DIETARY RESISTANT STARCH CONTENT ON THE GUT MICROBIOTA OF PATIENTS WITH SYSTEMIC LUPUS ERYTHEMATOSUS AND ANTIPHOSPHOLIPID SYNDROME}

\begin{abstract}
1,2Iryna Kulyk, 'Marcia S Pereira, 'Sylvio Redanz, ${ }^{3}$ William E Ruff, ${ }^{4}$ Teri M Greiling, ${ }^{5}$ Carina Dehner, ${ }^{6}$ Odelya Pagovich, ${ }^{7}$ Daniel Zegarra Ruiz, ${ }^{8}$ Cassyanne L Aguiar, ${ }^{9}$ Doruk Erkan, ${ }^{1,3,10}$ Martin A Kriegel*. ${ }^{1}$ Department of Translational Rheumatology and Immunology, Institute for Musculoskeletal Medicine, University of Münster, Münster, Germany; ${ }^{2}$ Indiana University School of Medicine, Indianapolis, IN, USA; ${ }^{3}$ Department of Immunobiology, Yale University School of Medicine, New Haven, CT, USA; ${ }^{4}$ Department of Dermatology, Oregon Health and Science University, Portland, OR, USA; ${ }^{5}$ Department of Pathology, Washington University School of Medicine, St. Louis, MO, USA; ${ }^{6}$ Department of Genetic Medicine, Weill Cornell Medicine, New York, NY, USA; ${ }^{7}$ Memorial Sloan Kettering Cancer Center, New York, NY, USA; ${ }^{8}$ Department of Pediatric Rheumatology, Children's Hospital of The King's Daughters, Eastern Virginia Medical School, Norfolk, VA, USA; ${ }^{9}$ Barbara Volcker Center for Women and Rheumatic Diseases, Hospital for Special Surgery, Weill Cornell Medicine, New York, NY, USA; ${ }^{10}$ Section of Rheumatology and Clinical Immunology, Department of Internal Medicine D, University Hospital Münster, Münster, Germany
\end{abstract}

\subsection{6/lupus-2021-lupus21century.87}

Background Diets deficient in fibers are prevalent in modern societies and implicated in gut microbial dysbiosis contributing to the pathogenesis of chronic inflammatory disorders. ${ }^{1}$ A particular type of dietary fiber, resistant starch (RS) type 2, was shown to ameliorate disease in murine models of systemic lupus erythematosus (SLE). ${ }^{2}$ This effect was mediated by 